\title{
Correlación entre diferentes contaminantes atmosféricos de la Ciudad de México y el Área Metropolitana
}

\section{Correlation between different air pollutants in Mexico City and the Metropolitan Area}

\author{
Israel Gutiérrez González \\ Universidad Autónoma del Estado de México, México \\ igutierrezg@uaemex.mx \\ (D) https://orcid.org/0000-0003-2984-7654 \\ Dora Maria Calderón Nepamuceno \\ Universidad Autónoma del Estado de México, México \\ dmcalderonn@uaemex.mx \\ (D) https://orcid.org/0000-0002-4296-4049 \\ Doricela Gutierrez Cruz \\ Universidad Autónoma del Estado de México, México \\ dgutierrezcr@uaemex.mx \\ (1) https://orcid.org/0000-0003-2983-7982 \\ Eduardo Aquino Vergara \\ Universidad Autónoma del Estado de México, México \\ eaquinov91@gmail.com \\ (1) https://orcid.org/0000-0003-4509-7478
}

Recepción: 28 de noviembre de 2018

Aprobación: 10 de junio de 2019

\section{Resumen}

Se lleva a cabo un estudio de correlación estadística entre datos numéricos correspondientes a la concentración de las partículas suspendidas y a la concentración de gases contaminantes que son obtenidos de las mediciones registradas en la base de datos de la Red Automática de Monitoreo Atmosférico (RAMA) para la Ciudad de México y el Área Metropolitana. Se reporta una importante dependencia entre los pares de gases $\mathrm{NO}_{2}-\mathrm{NO}_{X}, \mathrm{NO}-\mathrm{NO}_{\mathrm{X}}, \mathrm{CO}-\mathrm{NO}, \mathrm{CO}-\mathrm{NO}_{\mathrm{X}}$ los pares de partículas $\mathrm{PM}_{2.5}-\mathrm{PM}_{10}, \mathrm{PM}_{\mathrm{CO}}-\mathrm{PM}_{10}$ cuando el estudio se realiza tomando como referencia una sola estación de monitoreo. Por otra parte, se encuentra una estrecha relación entre el ozono $\left(\mathrm{O}_{3}\right)$ de una estación con el de otra para diferentes pares de estaciones.

Palabras ClaVe: contaminantes atmosféricos, correlación estadística, $\mathrm{PM}_{2.5}, \mathrm{PM}_{10} \mathrm{SO}_{2}, \mathrm{CO}, \mathrm{CO}_{2}, \mathrm{NO}, \mathrm{NO}_{2}, \mathrm{O}_{3}, \mathrm{NO}_{\mathrm{X}}$.

\section{Abstract}

A statistical correlation study is carried out between numerical data corresponding to the concentration of the suspended particles and the concentration of polluting gases which are obtained from the measurements registered in the Automatic Atmospheric Monitoring Network database (RAMA) for Mexico City and the Metropolitan Area. An important dependency between the gas pairs $\mathrm{NO}_{2}-\mathrm{NO}_{\mathrm{X}}$, $\mathrm{NO}-\mathrm{NO}_{\mathrm{X}}, \mathrm{CO}-\mathrm{NO}, \mathrm{CO}-\mathrm{NO}_{\mathrm{X}}$ and the $\mathrm{PM}_{2.5}-\mathrm{PM}_{10}, \mathrm{PM}_{\mathrm{CO}}-\mathrm{PM}_{10}$ particle pairs is reported when the study is carried out with reference to a single monitoring station. On the other hand, there is a close relationship between ozone $\left(\mathrm{O}_{3}\right)$ from one station with ozone to another for different pairs of stations.

KEYWORDS: Atmospheric pollutants, statistical correlation, suspended particles, polluting gases.

\section{INTRODUCCIÓN}

Los contaminantes atmosféricos se presentan como uno de los principales problemas por resolver en las grandes ciudades del mundo, por lo que los gobiernos han centrado sus esfuerzos y una buena cantidad de recursos para 
encontrar la fórmula que permita disminuir los efectos derivados de una mala calidad del aire sobre los seres vivos. Los inconvenientes de salud pública asociados con la contaminación ambiental han aumentado de forma paulatina y alarmante en las grandes urbes; las enfermedades que son constantes en la población van desde padecimientos leves en vías respiratorias o disminución de las capacidades de las funciones pulmonares hasta enfermedades mortales (OMS-OMM, 2012). Diversos estudios han encontrado que existe una correlación importante entre la contaminación del aire y la mortalidad por enfermedades respiratorias y cardiovasculares; en particular, algunos se han realizado tomando como base el aire de la Ciudad de México (Cohen et al., 1997; Borja et al., 1997, Borja et al., 1998; Gouveia y Leite-Junger (2017); Arceo et al. (2016); Calderón-Garcidueñas et al. (2016). Asimismo, y no menos importante, son notorios los efectos en los ecosistemas terrestres tanto en la vegetación como en el suelo tales como la reducción de la fotosíntesis, con efectos directos en el crecimiento de las plantas, reducción de su florecimiento y disminución en sus procesos reproductivos (Baird et al., 1996; Ramírez y Mendoza, 2008). Por otra parte, los ecosistemas acuáticos resultan afectados por la precipitación pluvial que se mezcla con todo tipo de contaminantes atmosféricos; se ha demostrado, por ejemplo, a) que los peces acumulan pesticidas en mayores cantidades que las plantas y $b$ ) que la lluvia ácida al entrar en contacto con las poblaciones de peces en cuerpos de agua y lagos propicia su reducción o desaparición (Environmental Protection Agency, 2004).

Otros aspectos donde se observa el impacto que tienen los contaminantes atmosféricos es en el cambio climático, en el deterioro de edificios y construcciones y en la reducción de la visibilidad, etc. Entre los principales contaminantes se encuentran los gases formados por los compuestos $\mathrm{SO}_{2}, \mathrm{CO}, \mathrm{CO}_{2}, \mathrm{NO}, \mathrm{NO}_{2}, \mathrm{O}_{3}, \mathrm{NO}_{\mathrm{X}}$ (dióxido de azufre, monóxido de carbono, dióxido de carbono, dióxido de nitrógeno, ozono y óxidos de nitrógeno respectivamente), así como las partículas $\mathrm{PM}_{2.5}$ (tamaño menor de $2.5 \mu \mathrm{m}$ ) y $\mathrm{PM}_{10}$ (tamaño menor que $10 \mu \mathrm{m}$ ), que dentro de sus componentes pueden presentarse varios elementos (plomo, hierro, níquel, cobre, platino, cromo, sodio, carbón) y otros compuestos orgánicos. Diferentes estudios de análisis estadístico de datos han determinado la correlación existente entre los factores que influyen en los índices de contaminación del ambiente y los distintos tipos de partículas y gases. Entre estos estudios podemos citar los análisis que se realizan entre contaminantes atmosféricos (partículas y gases) y variables meteorológicas (humedad, radiación solar, temperatura), donde los resultados evidencian que existe una importante correlación entre ambos tipos de datos y permiten saber que los factores meteorológicos inducen un aumento o disminución en la concentración de los principales contaminantes (Bravo et al., 2000; Amador et al., 2001).

Algunos estudios han demostrado la correlación entre partículas contaminantes pesadas y ligeras, lo cual permite entender la naturaleza de sus emisiones; si existe una correlación entre ambos tipos de partículas, se puede saber que la concentración y existencia de alguna de ellas depende de la concentración y existencia de otra. Al respecto, se encuentra el trabajo realizado por Echeverri y Maya (2008), quienes mediante una regresión lineal encontraron una correlación positiva entre las partículas $\mathrm{PM}_{2.5}$ y $\mathrm{PM}_{10}$ para algunas zonas cercanas a la ciudad de Medellín, Colombia. En este artículo, el análisis de la razón $\mathrm{PM}_{2.5} / \mathrm{PM}_{10}$ arroja como resultado que un porcentaje importante de partículas $\mathrm{PM}_{2.5}$ o partículas finas (> 60\%) se encuentra dentro del total de partículas respirables o $\mathrm{PM}_{10}$; lo anterior tiene una relación directa con la emisión de contaminantes debido a la combustión de combustibles según establecen Rojas y Galvis (2005).

Cabe resaltar que la acción conjunta de partículas suspendidas, gases contaminantes y otros compuestos pueden dar a lugar a una peligrosa sinergia, la cual se ha demostrado en diversos casos que tiene un impacto considerable en la salud y en el cuidado del ambiente. Como ejemplo de lo expuesto, está el trabajo de Pinkerton et al. (1989) que, tras un estudio realizado sobre ratas expuestas a ozono, muestra que la presencia de éste en el ambiente potenciaba la concentración pulmonar de amianto o asbesto, un agente que, se sabe, es cancerígeno. Este estudio se realizó a niveles bajos de ozono y se obtuvo una mayor retención pulmonar de las fibras de amianto inhaladas por los roedores. En otro estudio se comprueba un efecto sinérgico entre el $\mathrm{SO}_{2}$ y el ozono; en su reporte mensual de julio de 1999 de la Ley Canadiense de Protección Ambiental [CEPA] (1999), un estudio toxicológico establece que existen importantes efectos sobre la salud humana tras exposiciones de ozono seguidas de exposiciones a $\mathrm{SO}_{2}$, 
por lo que la interacción de los dos contaminantes puede potenciar los efectos negativos sobre la salud colectiva. Por otra parte, al ser el $\mathrm{SO}_{2}$ un gas bastante reactivo tiene la posibilidad de combinarse con otros contaminantes tales como los óxidos de nitrógeno $\mathrm{NO}_{X}$, los óxidos de carbono y el agua, combinación causante de la lluvia ácida, la cual perjudica de forma considerable los ecosistemas forestales y da lugar a la afectación y consecuente disminución paulatina de especies inmersas tanto animales como vegetales (Granados et al., 2010).

Tomando en cuenta los efectos que la combinación de distintos gases y partículas tienen sobre los seres vivos y el medioambiente, es importante seguir abordando este impacto sinérgico sobre todo en las grandes ciudades; de este modo, el objetivo del artículo es realizar un estudio estadístico de la correlación entre diferentes tipos de partículas y gases contaminantes en la Ciudad de México y el Área Metropolitana. Para este propósito, se consideran diversos tipos, de los cuales se tienen un conjunto de datos extraídos de las estaciones de monitoreo de concentración de contaminantes instaladas en diferentes puntos estratégicos de la Ciudad de México y Área Metropolitana. La teoría utilizada para la realización del artículo está basada en el cálculo de la correlación de Pearson para dos variables o atributos de un conjunto de datos. Se consideran seis diferentes gases y tres diferentes tipos de partículas, los cuales son de los contaminantes más estudiados. Sobre estos compuestos se calcula la correlación para la misma partícula o gas entre distintas estaciones y la correlación entre diferentes partículas o gases para la misma estación. Los resultados obtenidos proporcionan algunos patrones de comportamiento de acción conjunta entre contaminantes que pueden ser sometidos a análisis para continuar la investigación sobre los posibles efectos sinérgicos.

\section{Materiales y Métodos}

Para este estudio se tomó el conjunto de datos correspondientes a las concentraciones de gases y partículas contaminantes medidas durante 2017, provenientes de las estaciones del Sistema de Monitoreo Atmosférico, el cual se encuentra a cargo de la Secretaría del Medio Ambiente de la Ciudad de México. Estas bases de datos se encuentran recogidas en la Red RAMA.

Para estimar la relación estadística entre dos variables cuantitativas A y B, hacemos uso del coeficiente de correlación de Pearson -una técnica utilizada en minería de datos- definido por la siguiente expresión:

$$
r_{A, B}=\frac{\sum_{i=1}^{n}\left(a_{i}-\overline{A)}\left(b_{i}-\bar{B}\right)\right.}{n \sigma_{A} \sigma_{B}}
$$

donde $a_{i}$ y $b_{i}$ son los datos i-ésimos, $\bar{A}$ y $\bar{B}$ son los valores promedio y $\sigma_{A}$ y $\sigma_{B}$ son los valores de la desviación estándar para los atributos $\mathrm{A}$ y $\mathrm{B}$ respectivamente y $n$ es el número total de datos. En general, valores de $r_{A, B}$ cercanos a 1 y -1 corresponden a una correlación positiva o negativa, lo que significa que existe una dependencia relativa entre los dos tipos de datos A y B; la dependencia es relativa, ya que un valor de $r_{A, B}=1(0-1)$ corresponde a una nula dispersión en los datos, y a su vez corresponde al caso ideal. Valores cercanos a 0 para $r_{A, B}$, corresponden a una nula relación entre las variables $\mathrm{A}$ y $\mathrm{B}$ o una muy grande dispersión de los datos. Para establecer una interpretación del valor obtenido de $r_{A, B}$ y ver si los datos A y B están relacionados, y dicha relación no es producto del azar, se calcula el valor de la distribución $t$ de Student mediante la expresión:

$$
t=\frac{r_{A, B}}{\sqrt{\frac{1-r_{A, B}{ }^{2}}{n-2}}}
$$

y se compara con el valor obtenido de las tablas de $t$ de Student para un cierto nivel de significancia $\alpha$ (se ha establecido que $\alpha=0.05$ corresponde a un resultado aceptable). Los criterios que se utilizan son $a$ ) si la $t$ de Student obtenida de las tablas es menor que la calculada, entonces se dice que las variables están relacionadas; la 
correlación obtenida no proviene del azar (se rechaza hipótesis nula). b) Si la $t$ obtenida de las tablas es mayor que la calculada, entonces, se dice que no hay correlación entre las variables, aunque el valor de $r_{A, B}$ sea distinto de 0 (se acepta hipótesis nula).

\section{Desarkollo}

Para el cálculo del coeficiente de correlación $r_{A, B}$, nuestros atributos A y B corresponden a las mediciones que se hacen sobre las concentraciones de las partículas y gases contaminantes en intervalos de una hora durante un mes. Para este estudio se consideran las mediciones de las concentraciones de los gases dióxido de azufre $\left(\mathrm{SO}_{2}\right)$, dióxido de nitrógeno $\left(\mathrm{NO}_{2}\right)$, monóxido de carbono $(\mathrm{CO})$, óxido nítrico $(\mathrm{NO})$, óxidos de nitrógeno $\left(\mathrm{NO}_{\mathrm{X}}\right)$, ozono $\left(\mathrm{O}_{3}\right)$ y las partículas $\mathrm{PM}_{2.5}, \mathrm{PM}_{10}$ y $\mathrm{PM}_{\mathrm{CO}}$ (estas últimas llamadas fracción gruesa, coars, de las partículas $\mathrm{PM}_{10}$ y cuyo tamaño se encuentra entre $2.5 \mu \mathrm{m}$ y $10 \mu \mathrm{m}$ ) durante 2017 . El estudio se realizó con los datos obtenidos de 10 estaciones que se eligieron de acuerdo con su distribución geográfica como muestra representativa de las 43 estaciones (mapa 1).

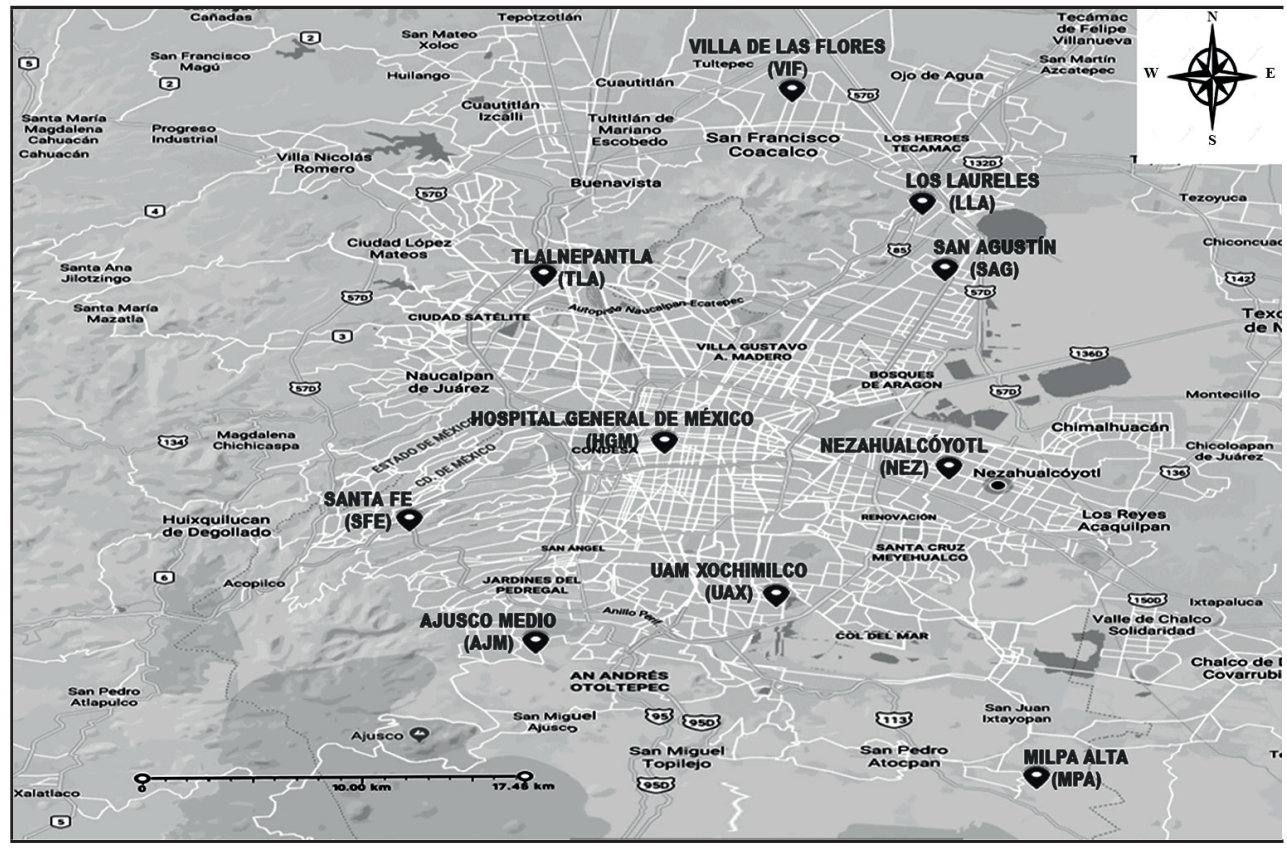

MAPA 1

Estaciones de monitoreo de emisión de partículas y gases contaminantes cuyos datos se analizan en este trabajo

Fuente: Google Maps (2019).

Las 10 estaciones están ubicadas en el norte, sur, poniente y oriente de la Ciudad de México a distancias considerables y se encuentran distribuidas tanto en sus alcaldías como en el Área Metropolitana (Estado de México) y tienen por nombre: Ajusco Medio (AJM), Hospital General (HGM), Los Laureles (LLA), Milpa Alta (MPA), Nezahualcóyotl (NEZ), San Agustín (SAG), Santa Fe (SFE), Tlalnepantla (TLA), UAM Xochimilco (UAX) y Villa de las Flores (VIF). Por cada mes, se tiene un total de hasta 744 datos por contaminante; los equipos de medición de algunas estaciones arrojaron datos que consideramos erróneos (números negativos), por lo que se realizó una limpieza de los datos. En la primera fase del estudio se calcula el coeficiente de correlación entre distintas partículas y gases para una misma estación durante cada mes desde enero de 2017 hasta diciembre de 2017, es decir, se eligen dos contaminantes (A y B) y las distintas combinaciones entre ellos: gas-gas, gas-partícula, partícula-partícula. 


\section{Resultados Y Discusión}

Para la estación de Ajusco Medio, se observan los pares de contaminantes en donde se tiene una alta correlación (>0.8), así como los meses a los que corresponden para las distintas combinaciones entre gases y partículas y en donde se verifica que la correlación de las variables no está determinada por el azar de acuerdo con los criterios mencionados en la metodología (cuadro 1).

\section{CUADRO 1}

Pares de datos de partículas y gases contaminantes para los cuales se obtuvo un coeficiente de correlación mayor a 0.8 y mes al que corresponden para la estación Ajusco Medio

\begin{tabular}{llll}
\hline Gas o Partícula $\mathrm{A}$ & Gas o Partícula B & \multicolumn{1}{c}{ Mes } & $r_{A, B}$ \\
\hline $\mathrm{NO}_{2}$ & $\mathrm{NO}_{\mathrm{X}}$ & Enero & 0.89 \\
$\mathrm{NO}$ & $\mathrm{NO}_{\mathrm{X}}$ & Enero & 0.86 \\
$\mathrm{PM}_{10}$ & $\mathrm{PM}_{2.5}$ & Enero & 0.9 \\
$\mathrm{NO}_{2}$ & $\mathrm{NO}_{\mathrm{X}}$ & Febrero & 0.94 \\
$\mathrm{NO}$ & $\mathrm{NO}_{\mathrm{X}}$ & Febrero & 0.85 \\
$\mathrm{PM}_{10}$ & $\mathrm{PM}_{\mathrm{CO}}$ & Febrero & 0.88 \\
$\mathrm{NO}$ & $\mathrm{NO}_{\mathrm{X}}$ & Marzo & 0.85 \\
$\mathrm{NO}_{2}$ & $\mathrm{NO}_{\mathrm{X}}$ & Abril & 0.96 \\
$\mathrm{NO}$ & $\mathrm{NO}_{\mathrm{X}}$ & Abril & 0.86 \\
$\mathrm{PM}_{10}$ & $\mathrm{PM}_{2.5}$ & Abril & 0.85 \\
$\mathrm{PM}_{10}$ & $\mathrm{PM}_{\mathrm{CO}}$ & Abril & 0.86 \\
$\mathrm{PM}_{10}$ & $\mathrm{PM}_{\mathrm{CO}}$ & Mayo & 0.84 \\
$\mathrm{NO}_{2}$ & $\mathrm{NO}_{\mathrm{X}}$ & Junio & 0.91 \\
$\mathrm{PM}_{10}$ & $\mathrm{PM}_{2.5}$ & Julio & 0.94 \\
$\mathrm{NO}_{2}$ & $\mathrm{NO}_{\mathrm{X}}$ & Agosto & 0.89 \\
$\mathrm{NO}$ & $\mathrm{NO}_{\mathrm{X}}$ & Agosto & 0.85 \\
$\mathrm{PM}_{10}$ & $\mathrm{PM}_{2.5}$ & Agosto & 0.93 \\
$\mathrm{NO}_{20}$ & $\mathrm{NO}_{\mathrm{X}}$ & Septiembre & 0.89 \\
$\mathrm{PM}_{10}$ & $\mathrm{PM}_{2.5}$ & Septiembre & 0.94 \\
$\mathrm{PM}_{10}$ & $\mathrm{PM}_{\mathrm{CO}}$ & Septiembre & 0.87 \\
$\mathrm{PM}_{10}$ & $\mathrm{PM}_{2.5}$ & Octubre & 0.93 \\
$\mathrm{PM}_{10}$ & $\mathrm{PM}_{\mathrm{CO}}$ & Octubre & 0.84 \\
$\mathrm{NO}_{2}$ & $\mathrm{NO}_{\mathrm{X}}$ & Noviembre & 0.87 \\
$\mathrm{PM}_{10}$ & $\mathrm{PM}_{2.5}$ & Noviembre & 0.86 \\
$\mathrm{PM}_{10}$ & Diciembre & 0.93 \\
$\mathrm{PM}_{10}$ & Diciembre & 0.87 \\
\hline
\end{tabular}

Fuente: elaboración propia basado de Sistema de Monitoreo Atmosférico de la CDMX (2018). 
Con base en estos datos, y para esta estación, podemos contar el número de veces que se tuvo una correlación mayor a 0.8 entre distintos tipos de partículas y gases, lo cual permite saber qué pares estuvieron más correlacionados: el par $\mathrm{NO}_{2}-\mathrm{NO}_{X}$ se correlacionó siete veces (siete meses) durante el año, el par $\mathrm{NO}-\mathrm{NO}_{X}$ cinco veces, el par $\mathrm{PM}_{2.5}-\mathrm{PM}_{10}$ ocho veces y el par $\mathrm{PM}_{\mathrm{CO}}-\mathrm{PM}_{10}$ seis veces durante el año cuando se realizó el estudio, las demás combinaciones no presentaron una correlación apreciable. En esta estación se observa que en los meses de febrero y abril se tuvo la más alta correlación, la cual corresponde al par $\mathrm{NO}_{\mathrm{X}}-\mathrm{NO}_{2}$. Lo anterior se debe a que, en este caso, el dióxido de nitrógeno destaca de entre los óxidos de nitrógeno $\mathrm{NO}_{X}$, recordando que el gas $\mathrm{NO}_{2}$ y el $\mathrm{NO}$ componen al $\mathrm{NO}_{X}$. En este sentido, durante agosto, septiembre y diciembre se tienen los valores más altos de correlación, que corresponden al par $\mathrm{PM}_{10}-\mathrm{PM}_{2.5}$, pues la combustión de hidrocarburos es uno de los orígenes de ambos tipos de partícula. En el cuadro 1 se distingue el conjunto de resultados para las 10 estaciones, en donde se resume la cantidad de veces que hubo una correlación apreciable por pares de partículas y gases de acuerdo con los criterios propuestos. Asimismo, en el cuadro 2 se aprecian sólo seis combinaciones distintas de partículas y gases donde se correlacionaron en al menos un mes: $\mathrm{NO}_{2}-\mathrm{NO}_{X}, \mathrm{NO}-\mathrm{NO}_{\mathrm{X}}, \mathrm{CO}-\mathrm{NO}, \mathrm{CO}-\mathrm{NO}_{\mathrm{X}}, \mathrm{PM}_{2.5}-\mathrm{PM}_{10}, \mathrm{PM}_{\mathrm{CO}}-\mathrm{PM}_{10}$. De esta forma, nos percatamos que los gases se correlacionan con los gases y las partículas con las partículas, y no se encontró correlación importante entre gases y partículas. Por su parte, los gases $\mathrm{SO}_{2}$ y $\mathrm{O}_{3}$ no presentan correlación con ningún otro gas o partícula. Es de notarse que la mayor cantidad de meses en la que dos pares de partículas o gases estuvieron correlacionados fue el par $\mathrm{NO}-\mathrm{NO}_{\mathrm{X}}$; particularmente, para las estaciones de HGM, SFE y UAX se registra un total de 10 meses en los que estos gases presentaron una correlación considerable. Esta alta correlación encontrada entre los óxidos de nitrógeno y el óxido nítrico es un elemento que sería de esperarse debido a que los óxidos de nitrógeno resultan de la combinación de los gases $\mathrm{NO}$ y $\mathrm{NO}_{2}$.

\section{CUADRO 2}

Conteo de número de meses en los que hay una correlación significativa entre pares de datos de partículas y gases contaminantes $\left(r_{A, B}>0.8\right)$ para las 10 estaciones

\begin{tabular}{|c|c|c|c|c|c|c|}
\hline Estación Pares & $\mathrm{NO}_{2}-\mathrm{NO}_{\mathrm{X}}$ & $\mathrm{NO}-\mathrm{NO}_{\mathrm{X}}$ & $\mathrm{CO}-\mathrm{NO}$ & $\mathrm{CO}-\mathrm{NO}_{\mathrm{X}}$ & $\mathrm{PM}_{2.5}-\mathrm{PM}_{10}$ & $\mathrm{PM}_{\mathrm{CO}}-\mathrm{PM} 10$ \\
\hline AJM & 7 & 5 & 0 & 0 & 8 & 6 \\
\hline HGM & 2 & 10 & 0 & 0 & 8 & 1 \\
\hline LLA & 0 & 0 & 0 & 0 & 0 & 0 \\
\hline MPA & 0 & 0 & 0 & 0 & 4 & 4 \\
\hline NEZ & 1 & 8 & 1 & 1 & 0 & 0 \\
\hline SAG & 0 & 9 & 1 & 2 & 1 & 5 \\
\hline SFE & 2 & 10 & 0 & 0 & 9 & 6 \\
\hline TLA & 0 & 8 & 2 & 2 & 6 & 8 \\
\hline UAX & 0 & 10 & 0 & 0 & 0 & 0 \\
\hline VIF & 2 & 9 & 0 & 6 & 0 & 0 \\
\hline
\end{tabular}

Fuente: elaboración propia.

Contrario a este comportamiento, los pares de gases que contienen al CO presentan pocos meses de correlación y en pocas estaciones; la estación VIF es la única en donde la mitad del año se tiene una correlación entre el CO y el $\mathrm{NO}_{\mathrm{X}}$. Por otra parte, las partículas $\mathrm{PM}_{10}$ presentan una importante correlación con las partículas $\mathrm{PM}_{2.5}$ y $\mathrm{PM}_{\mathrm{CO}}$ en distintas estaciones, por lo que la presencia de la $\mathrm{PM}_{10}$ se asocia de forma directa con la de la $\mathrm{PM}_{2.5}$ y de la $\mathrm{PM}_{\mathrm{CO}}$ y confirma el efecto sinérgico encontrado por Echeverri y Maya (2008) en la ciudad 
de Medellín. En el caso de la estación Los Laureles ubicada en Ecatepec, Estado de México, no se encontró correlación de ningún tipo. En la gráfica 1 se observa el comportamiento de los valores de concentración por pares de partículas o gases y en donde se advierte la dependencia que hay entre estos contaminantes; estas gráficas corresponden a aquellos pares de contaminantes donde se tienen los valores más altos calculados del coeficiente de correlación para cada una de las 10 estaciones. Se puede ver que la dispersión es menor cuando la correlación es más grande, lo cual verifica el cálculo correcto del coeficiente de correlación $r_{A, B}$. Se reportan nueve gráficos, ya que en el caso de la estación Los Laureles (LLA) no se encontró alguna correlación significativa.

Por otra parte, se realizó el cálculo del coeficiente $r_{A B}$ para las partículas o gases, pero de dos diferentes estaciones. De esta forma, se busca ver la relación entre la partícula o gas de alguna estación (atributo A) respecto a la partícula o gas de otra estación (atributo B). Se eligieron cinco pares de estaciones, cuya separación fuera mayor a $30 \mathrm{~km}$ de distancia y una estación con separación de $19 \mathrm{~km}$ de distancia. En el cuadro 3 se muestran los pares de estaciones, su distancia y el número de veces en el que las partículas o gases están correlacionadas con un coeficiente de correlación mayor a 0.8 . Es visible que en la mayoría de los casos la más alta correlación entre dos estaciones se da entre el gas $\mathrm{O}_{3}$ (ozono) de una estación respecto del gas $\mathrm{O}_{3}$ de otra; el mayor número de meses que están correlacionados por este gas entre dos distintas estaciones es de 11 , y corresponde $\mathrm{al}_{3}$ de la estación Los Laureles (LLA) y el $\mathrm{O}_{3}$ de la estación UAM-Xochimilco (UAX). En la combinación de las estaciones Villa de las Flores con San Agustín (VIG-SAG) se registraron correlaciones $\mathrm{NO}_{2}-\mathrm{NO}_{2}$, NO-NO y $\mathrm{NO}_{X}-\mathrm{NO}_{X}$, pero sólo en 2, 1 y 1 meses respectivamente; en todas las demás combinaciones posibles entre partículas y gases entre diferentes estaciones no se registró una correlación apreciable.

\section{CUADRO 3}

Distancia entre estaciones y conteo de número de meses en los que hay una correlación mayor a 0.8 entre pares de datos de partículas y gases contaminantes para dos estaciones distintas

\begin{tabular}{lccccc}
\hline Estaciones & Distancia de separación $(\mathrm{km})$ & $\mathrm{O}_{3}-\mathrm{O}_{3}$ & $\mathrm{NO}_{2}-\mathrm{NO}_{2}$ & $\mathrm{NO}-\mathrm{NO}$ & $\mathrm{NO}_{\mathrm{X}}-\mathrm{NO}_{\mathrm{X}}$ \\
\hline LLA-UAX & 31.6 & 11 & 0 & 0 & 0 \\
SAG-MPA & 37.9 & 1 & 0 & 0 & 8 \\
NEZ-SFE & 31.3 & 8 & 0 & 0 & 0 \\
TLA-AJM & 39.8 & 5 & 0 & 0 & 0 \\
VIF-NEZ & 35.4 & 9 & 0 & 0 & 0 \\
VIF-SAG & 19.0 & 10 & 2 & 1 & 1 \\
\hline
\end{tabular}

Fuente: elaboración propia.

La gráfica 2 muestra las gráficas de dispersión para dos diferentes estaciones para el caso de la relación $\mathrm{O}_{3}-\mathrm{O}_{3}$, donde se puede observar nuevamente la dependencia que existe entre la emisión que mide una estación respecto de la otra; se grafican únicamente los valores más altos de correlación que se obtuvieron.

En los resultados mostrados en el cuadro 3 también se puede ver que no existe correlación apreciable para todas las otras posibles combinaciones entre partículas y gases para los seis distintos pares de estaciones. La dependencia más importante se da con el ozono, y su caso más relevante se observa en el ozono de la estación UAX respecto al de la estación LLA donde se reportan 11 meses de alta correlación, a pesar de que la distancia entre las dos estaciones es grande. Esta relación conduce necesariamente a la tarea de revisar los datos de concentración de los óxidos de nitrógeno $\left(\mathrm{NO}_{\mathrm{X}}\right)$, los cuales, en acción conjunta con los compuestos orgánicos volátiles (COV), dan lugar al ozono troposférico (Aránguez et al., 1999; Clapp y Jenkin, 2001; Galán y Fernández, 2006). En el caso de los compuestos orgánicos volátiles como formadores de ozono, aunque no se estudian en este artículo, es importante no descartarlos de la investigación, ya que también se ha demostrado que tienen un impacto importante en el medioambiente (Ruiz et al., 1996) y en la salud pública (Jareño et al., 2017). 
a)

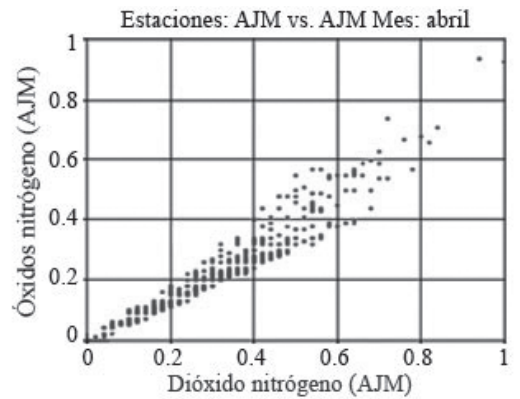

b)

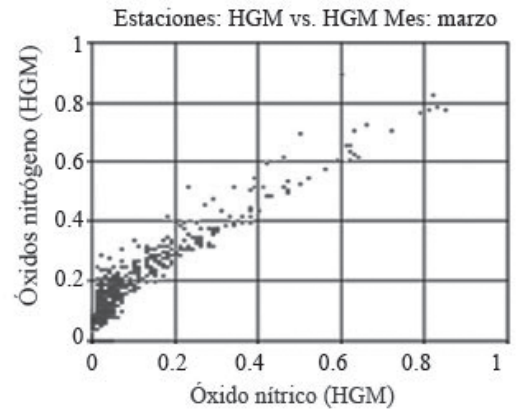

d)

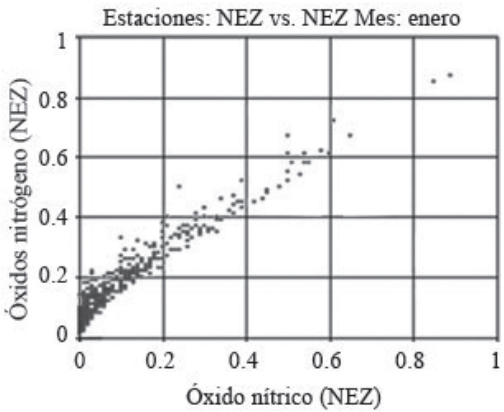

f)

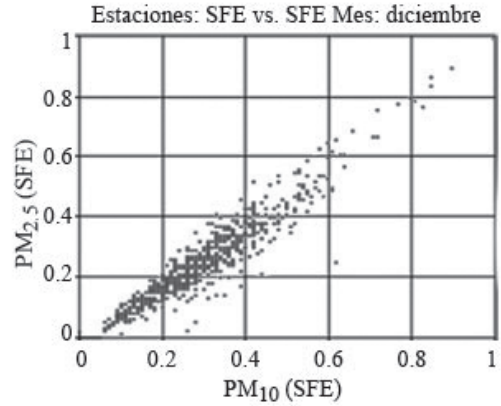

h)

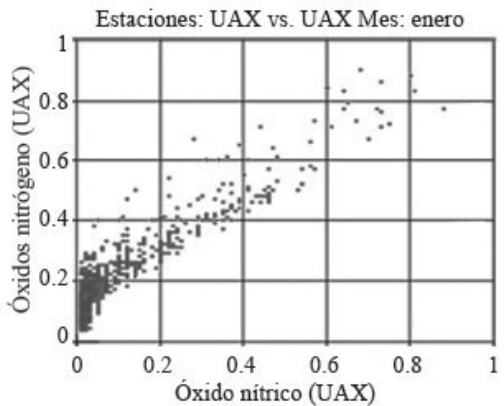

c)

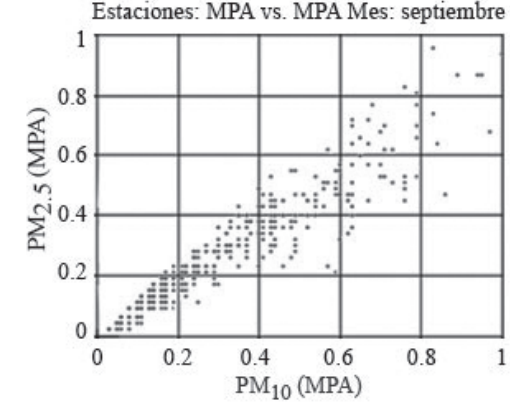

e)

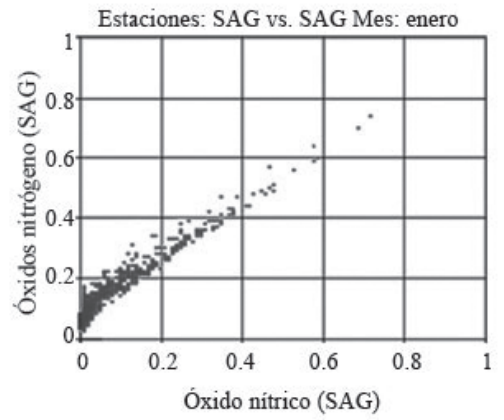

g)

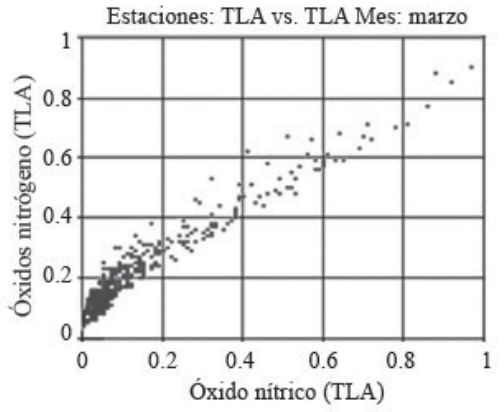

i)

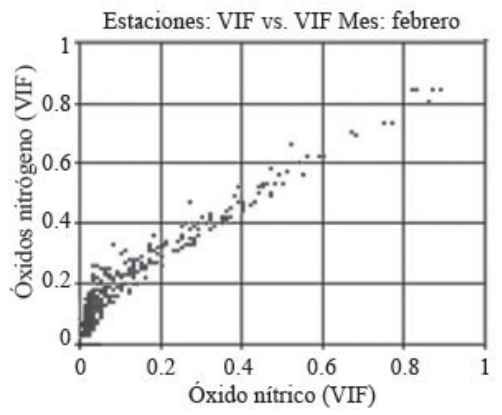

GRÁFICA 1

Gráficas de relación entre emisiones de contaminantes por pares de partículas o gases y por mes Fuente: elaboración propia. 
a)

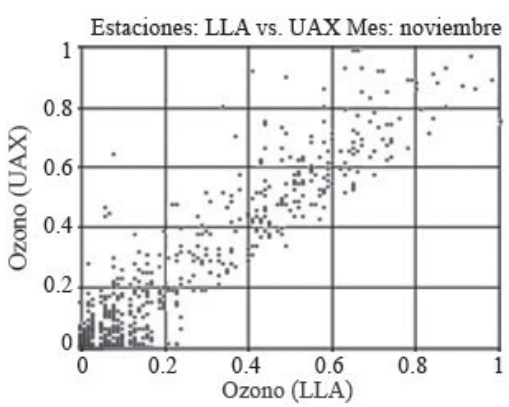

c)

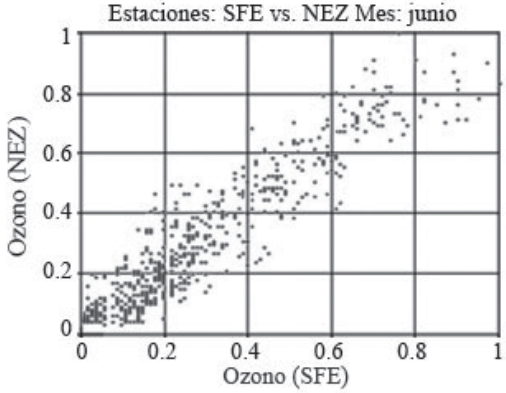

e)

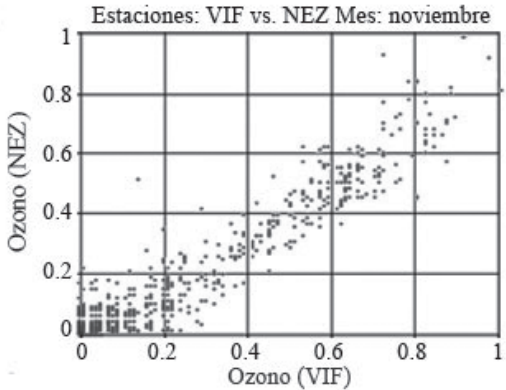

b)

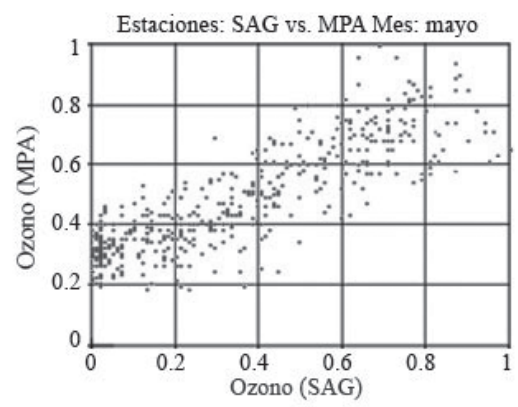

d)

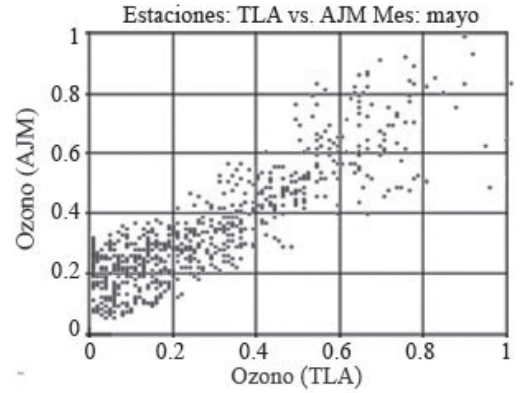

f)

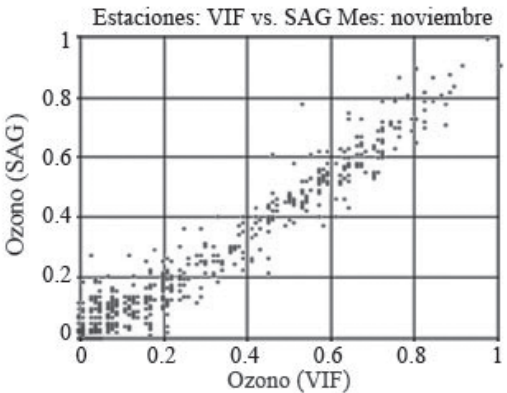

GRÁFICA 2

Gráficas de relación entre emisiones de ozono entre diferentes estaciones y por mes Fuente: elaboración propia.

Nota: a) Los Laureles-UAM Xochimilco (noviembre). b) San Agustín-Milpa Alta (mayo). c) Nezahualcóyotl-Santa Fe (junio).

d) Tlalnepantla-Ajusco Medio (mayo), e) Villa de las Flores-Nezahualcóyotl (noviembre), f) Villa de las Flores-San Agustín (noviembre).

\section{Análisis PROSPECTIVo}

Uno de los aspectos que pueden despertar un interés inmediato tanto para la investigación que se desarrolla en materia de medioambiente, así como aquella realizada en el campo de la minería de datos, es el conocimiento de la existencia de una tendencia en el comportamiento dinámico de los contaminantes atmosféricos; con el análisis propuesto en este trabajo -al conocer la correlación o dependencia de algún contaminante con respecto de otros- se debe estar en la posibilidad de reducir también los efectos sinérgicos a los que conducen. Desde la perspectiva de la salud, las investigaciones de actualidad refieren la importancia de continuar con el análisis de algunos contaminantes debido a sus potenciales efectos en la salud pública. Entre estos contaminantes se citan precisamente aquellos reportados en este artículo, los cuales destacan por su mayor correlación estadística. Las partículas $\mathrm{PM}_{2.5}$, para las que encontramos una alta correlación con las partículas $\mathrm{PM}_{10}$, son un importante y actual objeto de estudio en todo el mundo - particularmente en la Ciudad de México - tanto en el área de las ciencias exactas y aplicadas (Morton et al., 2018) como desde la perspectiva de la salud (Calderón et al., 2017; Quintana et al., 2018). Hernández et al. (2017) y Aguilar et al. (2018) coinciden en que el ozono, es 
un compuesto que sigue despertando el interés de la investigación en México. De acuerdo con los resultados encontrados, el conocimiento de que el ozono de alguna estación crece en proporción directa con el de otra estación -separadas en distancias considerables- es determinante para, primero, saber los lugares en donde es necesario poner atención con el objetivo de reducir las emisiones que provocan los altos índices de ozono y, segundo, extender el estudio a otros años para ver si el comportamiento que se encuentra en este trabajo se replica y de esta forma determinar si se establece una dependencia adicional de las concentraciones de ozono respecto a las estaciones del año (Barret y Raga, 2016).

\section{Conclusiones}

El estudio de las concentraciones de los principales gases y partículas suspendidas como contaminantes atmosféricos es una de las líneas de investigación más importantes en la actualidad debido a su constante amenaza sobre el medioambiente y los seres vivos. A través del cálculo de la correlación estadística entre dos variables distintas, este artículo busca determinar las posibles relaciones que existen entre estos contaminantes y encuentra que hay importantes efectos de dependencia entre algunos de ellos.

Al estudiar los datos obtenidos de las mediciones realizadas durante 2017 en algunas de las estaciones de monitoreo del sistema RAMA ubicadas en distintos puntos de la Ciudad de México y el Área Metropolitana, se encontró que existe una correlación significativa entre distintas partículas para una misma estación. Se reporta correlación entre los pares de gases y partículas $\mathrm{NO}_{2}-\mathrm{NO}_{X}, \mathrm{NO}-\mathrm{NO}_{\mathrm{X}}, \mathrm{CO}-\mathrm{NO}, \mathrm{CO}-\mathrm{NO}_{\mathrm{X}}, \mathrm{PM}_{2.5}-\mathrm{PM}_{10}, \mathrm{PM}_{\mathrm{CO}}-\mathrm{PM}_{10}$; esto significa que la concentración de algunos gases y partículas muestran una importante dependencia de la concentración de otros gases y partículas para una misma estación. En el caso de los compuestos con nitrógeno, la dependencia puede deberse a que el $\mathrm{NO}$ y el $\mathrm{NO}_{2}$ son dos de los compuestos que forman parte del total de óxidos de nitrógeno $\mathrm{NO}_{\mathrm{X}}$. El comportamiento de los pares de partículas PM se explica de forma similar, ya que las partículas $\mathrm{PM}_{2.5}$ y $\mathrm{PM}_{\mathrm{CO}}$ son parte del rango definido para las partículas $\mathrm{PM}_{10}$. No se encontró correlación entre gases con partículas suspendidas.

Otro resultado destacable es que se obtiene una importante correlación entre contaminantes del mismo tipo para diferentes estaciones. El caso más relevante es la estrecha relación obtenida entre el ozono de una estación respecto al de otra, como ejemplo sobresaliente se encuentra el par de Los Laureles y UAM-Xochimilco; en este proceso, las partículas suspendidas no participan en este tipo de correlación. Para analizar este comportamiento del ozono, y como trabajo a futuro, podría investigarse la forma como aumentan los óxidos de nitrógeno $\mathrm{NO}_{\mathrm{X}}$ y el monóxido de carbono $\mathrm{CO}$ durante noviembre (mes en el que se encontró la más alta de las correlaciones correspondiente a las estaciones Los Laureles y UAM-Xochimilco), ya que estos compuestos dan origen al ozono al reaccionar en la atmósfera en presencia de luz solar.

\section{Agradecimientos}

Se agradecen los comentarios de los árbitros de la revista que mejoraron sustancialmente el contenido del artículo.

\section{REFERENCIAS}

Aguilar, D., \& Reyes, I. (2018). A wavelet analysis of multiday extreme ozone and its precursors in Mexico City during 2015-2016. Atmospheric Environment, 188, 112-119.

Amador, O., Delgado, A., Villalobos, R., Munive, Z., Ortíz, R., Díaz, G., Bravo, J. L., \& Gómez, S. (2001). Partículas suspendidas, hidrocarburos aromáticos policíclicos y mutagenicidad en el Suroeste de la Ciudad de 
México. Contaminación Ambiental, 17(4), 193-204.

Aránguez, E., Ordóñez J. M., Serrano J., Aragonés, N., Fernández-Patier, R., Gandarillas A. y Galán I. (1999). Contaminantes atmosféricos y su vigilancia. Revista Española de Salud Pública, 73(3),421-423.

Arceo, E., Hanna, R., \& Oliva, P. (2016). Does the effect of pollution on infant mortality differ between developing and developed countries? Evidence from Mexico City. Economic Journal, 126(591), 257-280.

Baird, D. J., Maltby, L. Greig-Smith, P. W., \& Douben, P. E. T. (1996). Ecotoxicology: Ecological Dimensions. London: SETAC, Chapman \& Hall.

Barret, B. S., \& Raga, G. B. (2016). Variability of winter and summer surface ozone in Mexico City on the intraseasonal timescale. Atmospheric Chemistry and Physics, 16, 15359-15370.

Borja, V., Castillejos, M., Gold, D., Bierzwinski, S., \& Loomis, D. (1998). Mortality and ambient fine particles in Southwest Mexico City. Environmental Health Perspectives, 106(12), 855.

Borja, V., Loomis, D., Shrikant, P., Bangdiwala, C. M., Shy, C. M., \& Rascon Pacheco, R. (1997). Ozone, suspended particles and daily mortality in Mexico City. American Journal of Epidemiology, 4(3), 258-268.

Bravo, J. L., Nava, M. M., \& Muhlia, A. (2000). Relaciones entre la magnitud del valor máximo de ozono, la radiación solar y la temperatura de la Ciudad de México. Contaminación Ambiental, $16(2), 45-44$.

Calderón, L., González, L. O., Kulesza, R. J., Fech, T. M., Pérez, G., Jiménez, M. A., Soriano, R. E., Solorio, E., Miramontes, J. J., Gómez, A., Bernal, A. F., Mukherjee, P. S., Torres, R., Mills, P. C., Wilson, W. J., Pérez, B., \& D’Angiulli, A. (2017). Exposures to fine particulate matter $\left(\mathrm{PM}_{2.5}\right)$ and ozone above USA standards are associated with auditory brainstem dysmorphology and abnormal auditory brainstem evoked potentials in healthy young dogs. Environmental Research, 158, 324-332.

Calderón-Garcidueñas, L., Reynoso-Robles, R., Vargas-Martínez, J., Gómez-Maqueo-Chew, A., Pérez-Guillé, B., Mukherjee, P. S., Torres-Jardón, R., Perry, G., \& Gonzalez-Maciel, A. (2016). Prefrontal white matter pathology in air pollution exposed Mexico City young urbanites and their potential impact on neurovascular unit dysfunction and the development of Alzheimer's disease. Environmental Research, 146, 404-417

CEPA (Canada Environmental Protection). (1999, July). National ambient air quality objectives for ground-level ozone: Science Assessment Document. A report by the Federal-Provincial Working Group on Air Quality Objectives and Guidelines. Ontario.

Clapp, L., \& Jenkin, M. E. (2001). Analysis of the relationship between ambient levels of $\mathrm{O}_{3}, \mathrm{NO}_{2}$ and $\mathrm{NO}$ as a function of NOx in the UK. Atmospheric Environment, 35(6), 391-405.

Cohen, A. J., Arden, C., \& Speizer, F. E. (1997). Ambient air pollution as a risk factor for lung cancer. Salud Pública de México, 39(4),1-10.

Echeverri, C. y Maya, G. (2008). Relación entre las partículas finas $\left(\mathrm{PM}_{2.5}\right)$ y respirables $\left(\mathrm{PM}_{10}\right)$ en la ciudad de Medellín. Revista Ingenierias. Universidad de Medellín, 7(12), 23-42.

Environmental Protection Agency. (2004). Air quality criteria for particulate matter. EPA/600/P-99/002aF.

Galán, D. y Fernández, R. (2006). Implicación de los $\mathrm{NO}_{\mathrm{X}}$ en la química troposférica. Revista Electrónica de Medio Ambiente UCM, 2, 90-103.

Gouveia, N., \& Leite-Junger, W. (2017). Effects of air pollution on infant and children respiratory mortality in four large Latin-American cities. Environmental Pollution, 232(2017), 385-391.

Granados, D., López, G. F. y Hernández, M. A. (2010). La lluvia ácida y los ecosistemas forestales. Revista Chapingo. Serie Ciencias Forestales y del Ambiente, 16(2), 187-206. 
Hernández, I. Y., Clemitshaw, K. C., \& Mendoza, A. (2017). Observed trends in ground-level O $\mathrm{O}_{3}$ in Monterrey, Mexico, during 1993-2014: Comparison with Mexico City and Guadalajara. Atmospheric Chemistry and Physics, 17, 9163-9185.

Jareño, J., Muñoz, M., Gómez, O., Utrilla, S., Gutiérrez, C., Aguilar, A., Collado, L. y Callol, L. (2017). Estudio de 5 compuestos orgánicos volátiles en aire exhalado en la enfermedad pulmonar obstructiva crónica. Archivos de Bronconeumología, 53(5), 229-290.

Morton, O., Garza, R., Hernández-Álvarez, E., Amador-Muñoz, O., García-Arreola, M. E., Ordoñez-Godínez, S. L., Beramendi, L., Santos, G. L., \& Rosas, I. (2018). Recognition of the importance of geogenic sources in the content of metals in $\mathrm{PM}_{2.5}$ collected in the Mexico City Metropolitan Area. Environmental Monitoring and Assessment, 190(2), 83-101.

OMS-OMM (Organización Mundial de la Salud-Organización Meteorológica Mundial. (2012). Atlas de la salud y del clima. Organización Mundial de la Salud y Organización Meteorológica Mundial.

Pinkerton, K. E., Brody, A. R., Miller, F. J., \& Crapo, J. D. (1989). Exposure to low levels of ozone results in enchaced pulmonary retention of inhaled asbestos fibers. American Review of Respiratory Disease, 140(4), 1075-1081.

Quintana, R., Krais, M. A., Kourangi, B., Rosas, I., Mucs, D., López, R., Bergman, Å., \& Alfaro, E. (2018). Phthalate esters on urban airborne particles: levels in $\mathrm{PM}_{10}$ and $\mathrm{PM}_{2.5}$ from Mexico City and theoretical assessment of lung exposure. Environmental Research, 161, 439-445.

Ramírez, R. P., \& Mendoza, C. A. (2008). Ensayos toxicológicos para la evaluación de sustancias químicas en agua y suelo. La experiencia en México. México: Secretaría de Medio Ambiente y Recursos Naturales, Instituto Nacional de Ecología.

Rojas, N. y Galvis, B. (2005). Relación entre PM $_{2.5}$ y PM 10 en la ciudad de Bogotá. Revista de Ingeniería. Universidad de Los Andes, 22, 54-60.

Ruiz, M. E., Arriaga J. L. y García I. (1996). Determinación de compuestos orgánicos volátiles en la atmósfera de la Ciudad de México mediante el uso de sistemas ópticos y métodos convencionales. Atmósfera, 9, 119-135.

\section{BY-NC-ND}

